\title{
Improving Life of Self-Configuring Wireless Sensor Network using Clustering Mechanism
}

\author{
Dr. Md. Yusuf Mulge \\ Professor, CVR College of Engineering / CSE Department, Hyderabad, India. \\ Email: dryusufmulge@gmail.com
}

\begin{abstract}
Mobile Adhoc NETwork, popularly known as MANET, is one of the networks, which is dynamic in nature, due to the reason that the nodes are added and removed as per the need. Hence, the architecture of the network is changing continuously with reference to the addition or removal of the nodes. To acquire efficient functionality from the network, the network is set to configure automatically [7]. Another associated task in this dynamic network is the inclusion of new node to the network at any instance of time. Due to this the N/w size grows drastically, as a result of which, managing the nodes in the $n / w$ becomes tedious and the dynamic behavior of the $n / w$ becomes more critical [8]. In proposed and implemented work, I have designed an Intelligent Auto configuration (IAC) method in Mobile Adhoc NETworks, in which efficiency and reliability has improved significantly, compared to the traditional, Dynamic Address Allocation (DAA), in Mobile Adhoc networks. In this research work, the $n / w$ is divided into small segments called clusters and each cluster is controlled by a Cluster Head (CH), which will be defined under multiple parameters including Connectivity vector, Stability vector, and Trust vector. In this network, the overload and under load conditions are managed with the help of Wireless Sensor network, reconfigured for a particular Cluster [9]. The overload is the condition, where, the number of packets is beyond the capacity of network interface queue size and underload condition is that, the number of packets in the network is very less, so that, most of the time the network is idle. In the proposed work I have used Matlab for its implementation.
\end{abstract}

\section{Index Terms: MANET, auto Configuration, WSN, Cluster.}

\section{INTRODUCTION}

The MANET, consists of independent (autonomous) computers, referred as nodes, in which each one collaborates by forwarding the packets to/between each other, so that they communicate with outside range of direct wireless transmission. In the Mobile Adhoc NETwork, it is not necessary to have a centralized administration control or fixed network infrastructure as required in traditional networks [10]. At any instance of time the information access point can be created quickly and inexpensively as needed. Due to wireless links, the communication in MANETS is slow as compared to wired links.

The nodes in this type of network handles the network activities such as topology recognition and message transfer. Thus, the mobile nodes themselves handle the routing function.
One of the important characteristics of this work is that, there is no need to configure the network, because, the network configures itself automatically. All the nodes whether, routers, host or simple node auto-configures themselves [2], which are connected through wireless links. The nodes which function as routers are free to move from one cluster to other cluster or even from one N/w to other N/w. Any node which wants to transfer message can do so only if it has a valid policy token and the required capability. As the network is connectionless oriented (i.e. wireless), in any random way, as per the requirement, new nodes may be added, or existing unrequired passive nodes may be removed from the network as a result of which network architecture changes continuously in an unpredictable level. The MANET can work in a standalone fashion or it can be connected to other N/ws (small or large), to coordinate the functioning of the entire merged network [3].

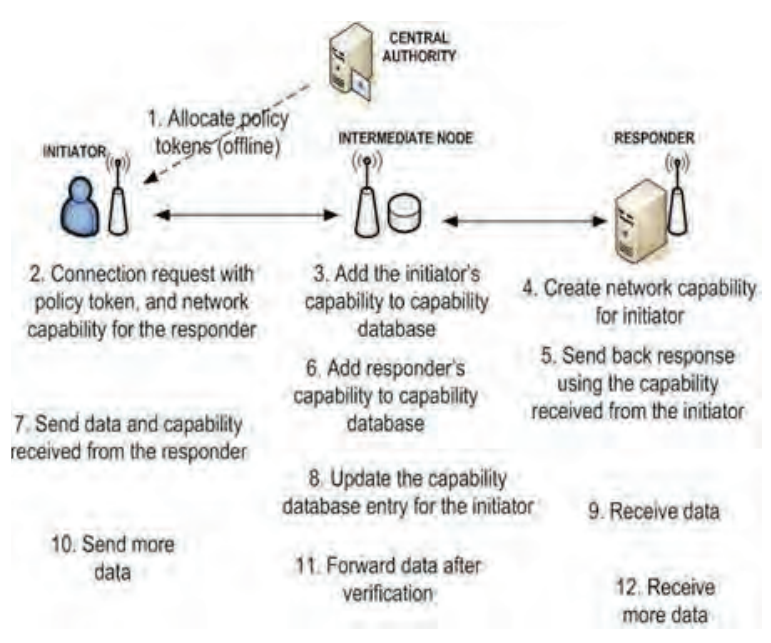

Figure 1. MANET System Architecture

Figure 1 depicts the architecture of Mobile ad-hoc N/w, which has one or more nodes acting as Group Controller (GC). The function of Group Controller is to allocate the required resources to the nodes during communication in the network. The allocation of resources is an important parameter and is allocated in the form of Policy Token. The policy tokens are signed by Group Controller and 
the signature is in encrypted form and can be verified by any node in the network. if a node has some data to send to other node, then the transmitting node(initiator) requests the receiver node(responder) to provide its service. The receiver node then sends back the capability to the transmitting node. This is called as Network Capability.

The major attributes of MANETs are as follows:

-Connectionless oriented communication.

-The role of Hosts and Routers Performed by nodes.

-The Bandwidth Constraints and Varying Capacity.

-Limited physical security.

-Dynamic Network Topology.

The following section discusses the properties of Mobile Adhoc NETwork.

\subsection{Properties of Mobile Adhoc NETwork routing protocols}

In the following section the properties are discussed in detail pertaining to MANETs.

1.1.1) Distributed operation: The protocol must be distributed. It should be independent of centralized node. This distributed operation gives the nodes the capability of entering or leaving the network as and when required, due to which the network changes and is required to be partitioned or merged as per the requirement.

1.1.2) Loopless network: The N/w performance can be improved, if the routing protocol assures, the routes to be free from loops. This ensures the efficient utilization of bandwidth or CPU capability [12].

1.1.3) On Demand Operation: The network has to manage overhead for resources and other control mechanisms, so to keep the control overhead within permissible limits in the network to ensure the efficient utilization of the network resources, the protocol should be active all the time and should respond only when required.

1.1.4) Unidirectional link: Due to high frequency signal in the ad-hoc networks, it uses unidirectional links. Due to unidirectional links, in which, data flows in only one direction at a time, throughput will enhance in the network.

1.1.5) Security: Any time there may be attack on the network from unreliable sources, due to the presence of high frequency signals, called as radio frequency signals. To provide security from this type of attacks the network should use security for routing. The ways to provide security are, one is 'Authentication' and the other means is 'Encryption'.

1.1.6) Multi-directional routes: To decrease the congestion in the network, the use of multiple routes is recommended. This is necessary because, the network is dynamic, in which the nodes get added and removed as per the need. This dynamically changes the topology and size of the network.

\subsection{Problems in routing with MANET}

1.2.1) Dissimilar links: Many of the connection oriented networks depends upon symmetric links that are fixed i.e they are static in nature. But in Mobile Adhoc NETworks the nodes are not fixed in the network. They change their position/cluster as and when required. Hence the links have dissimilar architecture.

1.2.2) Network-overhead: In Mobile Adhoc NETwork, due to continuous changing network topology there are always some stale routes that are preserved in the routing table. These stale routes are required to be deleted, so that they do not lead to wrong routes (and new routes are required to established). This leads to Routing Overhead.

1.2.3) Interference: Due to the presence of number of channels, the interference is the major problem in the wireless links.

1.2.4) Dynamic Topology: As the nodes are inserted and removed in the $\mathrm{N} / \mathrm{w}$ frequently, the topology keeps on changing continuously. So, Routing tables in the N/w must get updated frequently as and when required and the routing algorithms have to adapt to these changes.

\section{EXISTING WORK}

In the existing scheme called, Dynamic Address Allocation, the process of auto-configuration takes place as follows:

In wired networks each node is having an IP address, which is unique for each of the node. The IP address allocation is done by a server or a node acting as such which correctly assigns IP addresses. While, in mobile adhoc networks, there is no such centralized entity capable of carrying out this function. Therefore, a protocol is needed to perform the network configuration automatically and in a dynamic way, which will use all nodes in the network (or part thereof) as if they were servers that manage IP addresses. In my work one such most recently developed protocol called as D2HCP (Dynamic Host Configuration Protocol), is used for autoconfiguration.

In the existing scheme, the Adhoc NETwork is consisting of clusters and within each cluster, cluster-head is playing a vital role, which is responsible for managing the resources in the entire network [11]. As, the network in Mobile Adhoc NETwork is dynamic, the size of the network is continuously changing. If the mobile network grows in size, then it is partitioned into smaller networks. On the other hand, if the network shrinks then the number of such networks are merged. Under such circumstances each node is required to be assigned the HOST ID and MANET ID on the basis of their base value. There are mainly two techniques for merging; one is strong merging and the other is the weak merging technique. Table 1 shows the observations for these two merging techniques. 
TABLE I.

STRONG AND WEAK MERGING TECHNIQUES

\begin{tabular}{|c|c|c|c|c}
\hline S1.No & & Rounds & $\begin{array}{c}\text { Strong } \\
\text { Merging } \\
\text { Support }\end{array}$ & $\begin{array}{c}\text { Weak } \\
\text { Merging Support }\end{array}$ \\
\hline 01 & & 50 & 28 & 11 \\
\hline 02 & & 100 & 60 & 31 \\
\hline 03 & & 150 & 78 & 45 \\
\hline 04 & & 200 & 84 & 52 \\
\hline & & & & \\
\hline 05 & & 250 & 85 & 62 \\
\hline 06 & & 300 & 90 & 70 \\
\hline 07 & & 350 & 92 & 72 \\
\hline 08 & & 400 & 93 & 80 \\
\hline 09 & & 450 & 94 & 83 \\
\hline
\end{tabular}

Figure 2 plots the strong and weak merging techniques support, taking rounds on $\mathrm{x}$-axis and merging support on $\mathrm{y}$ axis. From the plot it is clear that strong merging technique supports more hops than weak merging technique.

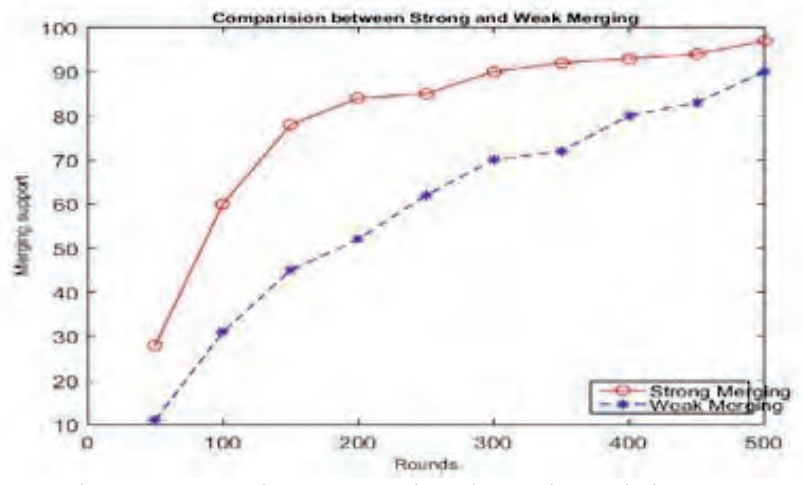

Figure 2. Comparing strong and weak merging techniques

In the traditional DAA, no hierarchy in the network is maintained, so if any of nodes become inactive during communication, then the whole network becomes useless. Further in this method no scheme is specified to include a new node in the network and no trust is defined.

Also, the Cluster Head does not monitor the overload and underload conditions.

Further auto-configuration improves performance over mobile network [13].

Finally, a multiple parameter approach is provided to configure the network.

\section{PROPOSED WORK}

The proposed work, Intelligent Auto Configuration (IAC) [14], is an improvement over the traditional Dynamic Address Allocation (DAA) in Mobile Adhoc networks, (and is presented with auto-configuration concept) [4]. Here the Wireless Sensor Network (WSN) is used. Based upon a number of parameters, the network configures itself, which is nothing but autoconfiguration. In traditional networks the reliability is achieved by retransmission of the lost packets. This scheme increases the overhead and also a lot of resources and time is consumed. To avoid this most of the WSNs use the scheme of redundancy by any one of the schemes, either hop-by-hop or end-to-end scheme. Hop-by-hop method allows the intermediate nodes to perform retransmission. On the other hand, end-to-end approach retransmission is performed only at the source and destination nodes. This way the efficiency and reliability increases.

The clustering mechanism used is "Mobility-aware clustering" such as, MOBIC. In this clustering mechanism nodes disseminate their mobility information (speed and direction). In this scheme, the node with lowest mobility is selected as Cluster-head. The work is defined as follows:

\subsection{Formation of Network with cluster mechanism}

At the initial stage, when the Wireless Sensor Network is configured, the analysis of network will be under the clustering mechanism [5]. Here the network will be divided into smaller partitions called clusters and each cluster is having cluster-center and cluster objects (Nodes) as cluster members. The cluster membership of a node will depend on three main parameters:

1. Connectivity vector

2. Trust vector

3. Stability vector.

One of the nodes will be selected as Cluster Head $(\mathrm{CH})$. Each cluster has an independent $\mathrm{CH}$. The $\mathrm{CH}$ is the one which has long association with that cluster or which has least mobility.

\subsection{To Handle Overload - underload conditions}

When clusters are formed, then the next task is monitoring these clusters to identify the overload and under load conditions. In case of overload, the cluster is divided into small clusters where as in the case of under load condition, clusters are merged to form a large cluster. The re-clustering mechanism and network configuration will be performed under such circumstances. This equalizes the distribution of nodes over the network as well as provide stability over the network [1].

\subsection{New Node Inclusion}

In the next step, the addition of a new node to the network will be done based on sequential-analysis algorithm.

\section{IMPLEMENTATION}

Figure 3 shows how the Mobile-Adhock NETwork is formed based on clustered mechanism.

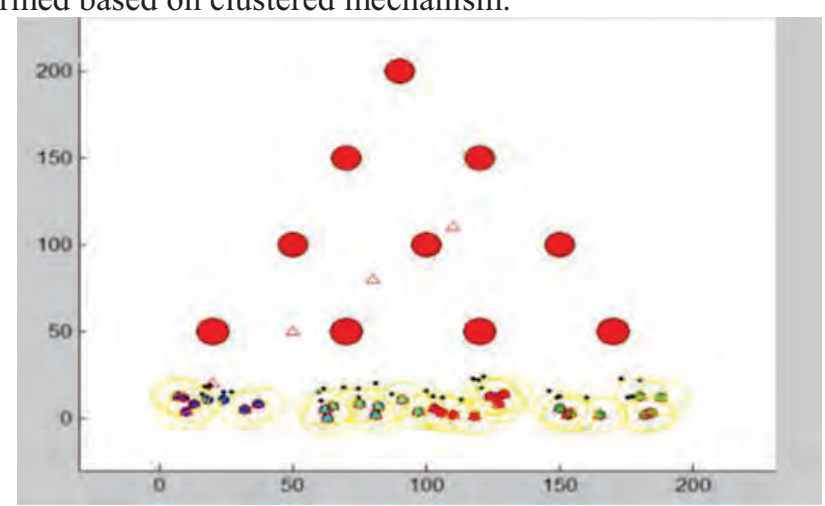

Figure 3. N/w configuration based on cluster 
The hierarchical N/w which is formed under clustered mechanism is used to describe the communication among all the nodes responsible for improvement of reliability and efficiency of the network [6]. The network has a base station. The nodes which are depicted in red color forms clusters and all other nodes participate in communication in the network.

Table 2 shows Dead Nodes in a WSN in Existing Versus Proposed implemented work.

TABLE II.

DEAD Nodes IN EXISTING Work VS Proposed Work

\begin{tabular}{|c|c|c|c|}
\hline Si.No. & Rounds & \multicolumn{2}{|c|}{ Dead Nodes } \\
\hline & & Existing Work & Proposed Work \\
\hline 01 & 25 & 20 & 0 \\
\hline 02 & 50 & 32 & 0 \\
\hline 03 & 75 & 37 & 17 \\
\hline 04 & 100 & 37 & 22 \\
\hline 05 & 200 & 38 & 27 \\
\hline 06 & 300 & 38 & 28 \\
\hline 07 & 400 & 38 & 31 \\
\hline 08 & 500 & 38 & 32 \\
\hline 09 & 600 & 38 & 35 \\
\hline 10 & 700 & 38 & 35 \\
\hline 11 & 800 & 38 & 37 \\
\hline 12 & 900 & 38 & 37 \\
\hline 13 & 1000 & 38 & 37 \\
\hline
\end{tabular}

Observations of table II are plotted in figure 4.

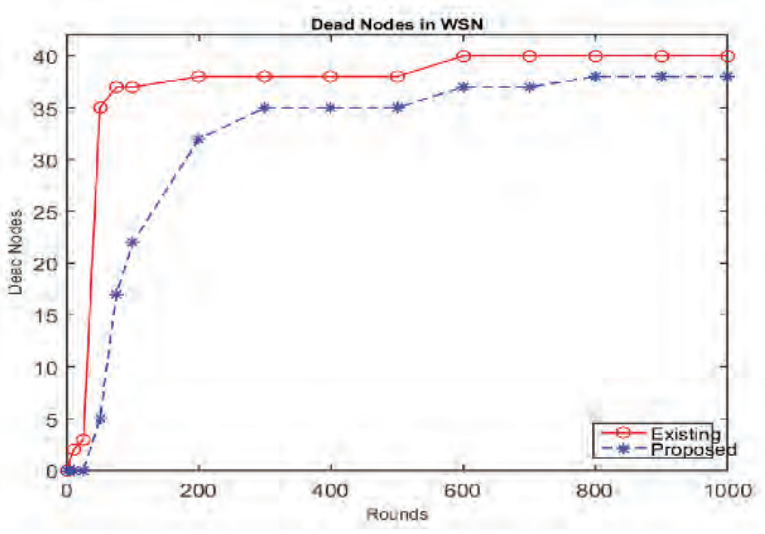

Figure 4. Nodes in WSN that are not active

Figure 4. Shows the analysis of dead nodes in both existing work (Blue curve) and proposed and implemented work (Green curve). It is clear from the figure, that the existing work require more energy and hence the life of the network is reduced. In contrast to this, the proposed implemented scheme increases the efficiency in terms of energy conservation and hence increases the life of the network.

Table 3 shows Active nodes in WSN in Existing Versus Proposed work, which is plotted in figure 5.
TABLE III.

ACTIVE NODES IN EXISTING VS PROPOSED WORK

\begin{tabular}{|c|c|c|c|}
\hline Si.No. & Rounds & \multicolumn{2}{|c|}{ Alive Nodes } \\
\hline & & \multicolumn{2}{|c|}{} \\
\hline & & $\begin{array}{c}\text { Existing } \\
\text { Work }\end{array}$ & $\begin{array}{c}\text { Proposed } \\
\text { Work }\end{array}$ \\
\hline 01 & 25 & 07 & 45 \\
\hline 02 & 50 & 07 & 28 \\
\hline 03 & 75 & 05 & 23 \\
\hline 04 & 100 & 05 & 22 \\
\hline 05 & 200 & 03 & 13 \\
\hline 06 & 300 & 02 & 09 \\
\hline 07 & 400 & 02 & 07 \\
\hline 08 & 500 & 02 & 07 \\
\hline 09 & 600 & 02 & 04 \\
\hline 10 & 700 & 02 & 04 \\
\hline 11 & 800 & 02 & 03 \\
\hline 12 & 900 & 02 & 03 \\
\hline 13 & 1000 & 02 & 03 \\
\hline & & & \\
\hline
\end{tabular}

Figure 5 Shows the number of alive nodes in Wireless Sensor Network in both proposed and existing work. It shows that network life remains up to 250 rounds in existing work whereas network life remains up to 800 rounds in the proposed implemented work.

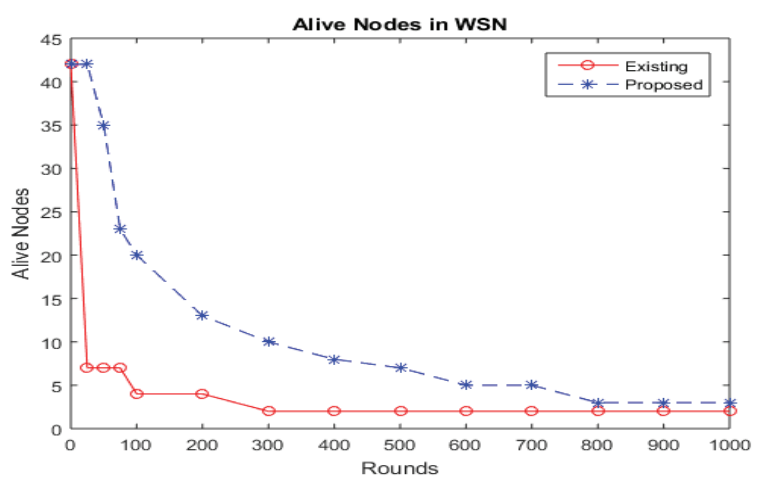

Figure 5. Active node in Wireless Sensor N/w

Table 4 shows Packets sent to Base Station in Existing Vs proposed work, which is plotted in figure 6 .

TABLE IV.

PACKets SENT To BASE StATION

\begin{tabular}{|c|c|c|c|c|}
\hline Si.No. & & Rounds & \multicolumn{2}{|c|}{ Packets Sent to Base Station } \\
\hline & & $\begin{array}{c}\text { Existing } \\
\text { work }\end{array}$ & $\begin{array}{c}\text { Proposed } \\
\text { work }\end{array}$ \\
\hline 01 & & 25 & 50 & 200 \\
\hline 02 & & 50 & 60 & 400 \\
\hline 03 & & 75 & 80 & 550 \\
\hline 04 & & 100 & 85 & 600 \\
\hline 05 & & 200 & 90 & 900 \\
\hline 06 & & 300 & 100 & 1200 \\
\hline 07 & & 400 & 100 & 1250 \\
\hline 08 & & 500 & 100 & 1300 \\
\hline 09 & & 600 & 100 & 1400 \\
\hline 10 & & 700 & 100 & 1500 \\
\hline 11 & & 800 & 100 & 1600 \\
\hline 12 & & 900 & 100 & 1650 \\
\hline 13 & & 1000 & 100 & 1700 \\
\hline
\end{tabular}


Figure 6 Shows the pkts transmitted to the BS (i.e. overall transmission to the Base Station over the network).

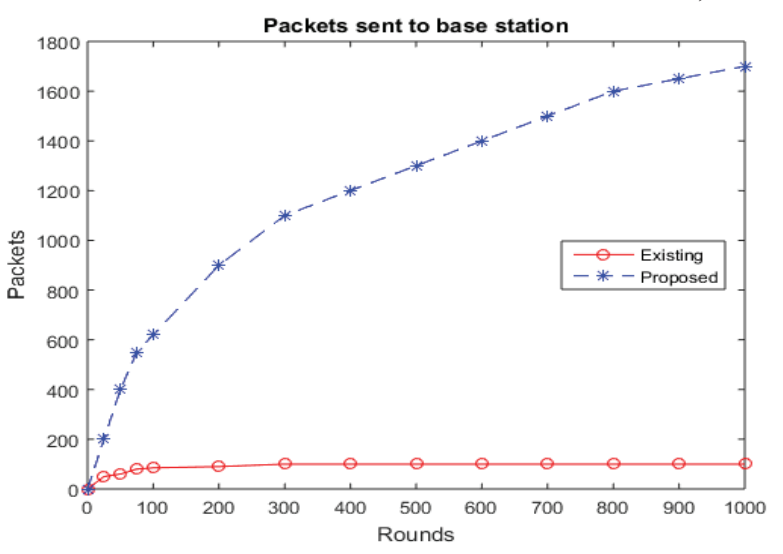

Figure 6. Pkts transmitted to the Base Station

From figure 6 it is clear that, the number of packets sent to the base station is more in implemented work, which increases the efficiency of transmission.

\section{CONCLUSions \& FUture WORK}

The proposed implemented Intelligent Auto configuration (IAC) scheme uses multiparametric N/w which is partitioned into clusters. Each cluster in the network consists of its own cluster head and the Cluster Head selection is based on trust vector so that network reliability can be improved. The N/W also handles the overload and under load situations in the network. In this work, a network can handle the inclusion of a node in level based approach in order to make network more effective. Further, one can improve network efficiency and reliability by performing the experiments with existing auto configuration protocols which includes the size adjustment of transmitted packet.

\section{REFERENCES}

[1] Amit Munjal, Yatindra Nath Singh, A. Krishna Phaneendra, Amitabha Roy, "Scalable Hierarchical Distributive AutoConfiguration Protocol for

MANET's". 2013 International Conference on SignalImage Technology\& Internet-Based Systems, pp 699-705.

[2] Ana Cavalli and Jean-Marie Orset, "Secure Hosts Autoconfiguration in Mobile Ad hoc Networks". Proceedings of the 24th International Conference on Distributed Computing Systems Workshops (C) 2004 IEEE,pp 1-6.

[3] M J Kim, M Kumar and B. A. Shirazi, "A Lightweight Scheme for Auto-configuration in Mobile Ad Hoc Networks". 19th IEEE International Parallel and Distributed Processing Symposium (IPDPS'05), pp 1-5.

[4] Mr. M. M. Iqbal, Dr. I. Gondal, Prof. L. Dooley, “Optimizing the Beacon Exchange Rate for Proactive Autonomic Configurationin Ubiquitous MANETs". (C) 2005 IEEE,pp 1-6.

[5] Mudasser Iqbal, Iqbal Gondal, Laurence S. Dooley, "Distributed and Load-Adaptive Self Configuration in Sensor Networks". 2005 Asia-Pacific Conference on Communications October 2005, pp 1-5.

[6] M. Nazeeruddin, Student Member, IEEE, G. P. Parr, Member, IEEE, and B. W. Scotney, "A New Stateful Host Autoconfiguration Protocol for Digital Battlefield MANET”, PP 17.
[7] Majid Taghiloo1, Jamshid Taghiloo, Mehdi Dehghan1, "A Survey of Secure Address Auto-Configuration in MANET". (C) 2006 IEEE, pp 1-5.

[8] Dong Shi, Xinming Zhang, Xuemei Gao, Wenbo Zhu, Fengfu Zou, "A Link Reliability-aware Route Maintenance Mechanism for Mobile Ad hoc Networks". Proceedings of the Sixth International Conference on Networking (ICN'07) C 2007 IEEE.

[9] Yang-Min Lee1, Bong-Soon Kang1, Jae-Kee Lee2, Hadan-2Dong,Saha-Gu, Busan, "A Study on a highly-reliable Multipath Configuration protocol in Ubiquitous Network by MANET".2007 International Conference on Multimedia and Ubiquitous Engineering (MUE'07) C 2007 IEEE.

[10] Zhang Ning, Sanmin Lee, Kiho Nam, Jongwan Kim, Jaepil Yoo,Kee-Cheon Kim, "Central Management and Tree Based Auto-configuration in MANET". International Conference on Advanced Computer Control (C) 2008 IEEE.

[11] Yang Yang, Jian Chen, Leiling Duan, Luoming Meng, Zhipeng Gao, Xuesong Qiu, "A Self-Configuration Management Model for Clustering-based MANETs". International conference on Networking, 2008.

[12] Ausama Yousef, Ali Diab and Andreas Mitschele-Thiel, "Performance Evaluation of Stateful Address AutoConfiguration Protocols in Ad hoc Network". C2009 IEEE.

[13] Nurul I. Sarkar, Wilford G. Lol, "A Study of MANET Routing Protocols: Joint Node Density, Packet Length and Mobility". (C)2010 IEEE.

[14] Vas aka Visoottiviseth, Chaiwat Yanprasop, Panita Pongpaibool. "DAA: Distributed Address Autoconfiguration for Mobile Ad Hoc Networks". Eighth International Joint Conference on Computer Science and Software Engineering (JCSSE) 2011, pp 1-6. 